\title{
Análisis de las finanzas personales de los estudiantes de la Corporación Universitaria Americana (CUA) sede Montería, respecto a la cultura del ahorro
} Analysis of the personal finances of the students of the American University Corporation (CUA) Montería headquarters, regarding the culture of savings

DOI: 10.21803/adgnosis.8.8.393

\section{Resumen}

Este proyecto investigativo se realizó tomando como muestra la población estudiantil de la Corporación Universitaria Americana, sede Montería, que se encuentra activa laboralmente, y se realizó un muestreo sobre manejo de sus finanzas y sus conocimientos sobre la cultura del ahorro. El objetivo principal fue analizar el comportamiento de las finanzas personales de los estudiantes universitarios que laboran de la CUA, Montería, para determinar qué proporción de esta población presenta la cultura del ahorro, para ello se contó con instrumentos de recolección como la encuesta, aplicandola la población estudiantil de la facultad de ciencias administrativas económicas y contables de la universidad que actualmente labora, arrojando como resultado el mayor porcentaje de la población inició a laborar entre los 15 y 20 años, tienen más de 5 años laborando, no tiene dependientes económicos.

Palabras clave: análisis, ahorro, finanzas, estudiantes, empleo

\section{Abstract}

This research project was carried out taking as a sample the student population of the Corporación Universitaria Americana-Montería headquarters, who are actively employed; a sample was carried out on the management of their finances and their knowledge about the culture of savings. The main objective was to analyze the behavior of the personal finances of the university students at CUA- Montería who work, to determine what proportion of this population presents the culture of savings. For this purpose, we had a survey collection instrument that was applied to the student population of the University's Administrative, Economic and Accounting Science Faculty that is currently working, giving as a result that the highest percentage of the population started working between the ages of 15 and 20 , they have been working for more than 5 years and there are no economic dependents.

Keywords: analysis, savings, finances, students, employment.

\author{
María José Yepes Benítez ${ }^{1}$ \\ yepesmaria33@americana.edu.co
}

Melissa Torres Cermeño²

rodríguezmelissa79@americana.edu.co

\section{Adaluz Peréz Lugo 3 \\ perezada90@americana.edu.co}

Merjoury Ballesteros Herrera ${ }^{4}$

mherrera@americana.edu.co

Alexander Bravo Yepes 5

ayepes@americana.edu.co

\section{Cómo citar este artículo:}

Yepes, M., Torres, M., Pérez, A., Ballesteros, M. \& Bravo, A. (2019). Análisis de las finanzas personales de los estudiantes de la Corporación Universitaria Americana (CUA) sede Montería, respecto a la cultura del ahorro. $8(8)$, p. $141-147$

DOI: 10.21803/adgnosis.8.8.393

1Tecnologo en procesos contables, Corporación Universitaria Americana, Montería.

2 Tecnologo en procesos contables, Corporación Universitaria Americana, Montería.

3 Tecnologo en procesos contables, Corporación Universitaria Americana, Montería.

4 Ingeniera agroindustrial, administradora de empresas, Magíster en dirección estratégica. Docente investigador de la Corporación Universitaria Americana, Montería, Colombia.

5Contador público, MBA con énfasis en finanzas empresariales y doctorando en educación. Docente investigador de la Corporación Universitaria Americana, Montería, Colombia. 


\section{Introducción}

En la actualidad la palabra "finanzas" provoca en una gran mayoría de individuos -Especialmente en los jóvenes- todo tipo de reacciones precavidas acerca del uso adecuado del dinero. Se podría decir que incluso algunas personas tienen sus propias definiciones e interpretaciones acerca de ésta cuando se les pregunta. Algunos la consideran como la buena administración del dinero, otros opinan que es simplemente tomar buenas decisiones económicas con respecto a los ingresos que se generan.

Por consiguiente, encontrarinformación financieraen pleno siglo XXI es tan sencillo como abrir una cuenta de G-mail, hoy en día existen infinidades de revistan financieras que hablan sobre temas económicos, revista que hablan sobre inversión y ahorros, en los periódicos y noticieros existen secciones especializadas en temas financieros y gracias a internet esa información financiera puede estar disponible en herramientas o redes sociales como Facebook, YouTube, etc. Sin embargo, pese a que la información es asequible y está disponible muy fácilmente, cierta cantidad de personas desconoce casi por completo el adecuado manejo de las finanzas. Los autores Redy, Bruhn, M y Tan (2013) realizaron una encuesta Nacional de Capacidades Financieras en Colombia, donde se evidencia que las aptitudes ahorrativas del colombiano promedio, preexiste una incompatibilidad entre el comportamiento financiera que se espera y el comportamiento financiera real de las personas, es decir, que aunque exista una proporción de Colombianos que muestran preocupación por sus finanzas, los resultados de los encuestas demuestran que en la práctica diaria los colombianos en sí tiene pocos hábitos ahorrativos, pues solo el $41 \%$ ahorraba a futuro, el $23 \%$ sabía cuánto gastaba semanalmente y el $1 \%$ tenía dinero ahorrado para gastos imprevistos.

Considerando lo anterior, es normal prever que los jóvenes adultos también puedan estar en esos porcentajes, la educación financiera se basa en ejemplos y las costumbres ahorrativas se empiezan a adquirir desde temprano.

Uno de los primeros momentos en el cual el individuo empieza a tener contacto con el mundo financiero, es cuando empieza a generar ingresos y a gastar su dinero en las necesidades de primera mano o quizás en aquellas su- perficiales, desde ese momento entra en juego las llamadas finanzas personales, que es la relación que se tiene con el entorno y el uso del dinero que se genera. Es una etapa muy delicada, en donde se formará hábitos económicos que pueden llegar a ser positivos o negativos en un futuro. En Colombia, los jóvenes que tienen la oportunidad de ingresar a una educación superior, a menudo lo hacen con altos desconocimientos y vacíos la sobre educación financiera y hábitos de ahorro, según el periódico El portafolio (2016) a los jóvenes universitarios colombianos a menudo les cuesta identificar tópicos financieros básicos como tasas de intereses convenientes, tasas de interés simple, manejo de tarjetas de crédito y productos financieros que puede ofrecer un banco; quedándose así, solo con los conocimientos financieros que trajeron desde casa, exponiéndose a malas decisiones financieras y endeudamientos a edades tempranas.

Financieramente hablando, el mundo es cada vez más agresivo, los mercados económicos son cada más complicados y surgen la necesidad de formar individuos con capacidades financieras que ayuden a crear su propia estabilidad económica, empezando por los jóvenes.

Los estudiantes de la CUA Montería activos laboralmente son jóvenes, que en su gran mayoría oscilan entre las edades de 18 y 30 años, unos con más experiencia que otros, pero igualmente comparten las mismas cargas laborales y estudiantiles. Así, tanto el estudio y el trabajo en personas jóvenes a menudo se transforma en cargas negativas difíciles de balancear, la misma persona, tanto en su rol como estudiante y como trabajador está sometida a mucha presión y está comprobado científicamente que el estrés repercute en niveles bastante altos en personas jóvenes que estudian y trabajan (Méndez, 2015). A menudo cuando las personas manejan altos grados de estrés lo primero que buscan es romper la rutina y emplear métodos distractores que los relajen.

Sin embargo, esos métodos distractores pueden ser contraproducentes si se exceden demasiados yno se tiene en cuenta la variable del ahorro como factor influyente, más coloquialmente -plataquesegana, plataquesegasta-losjóvenesamenudonoentienden la reglabásica deno exceder más de loque se gana, especialmente si susingresosnoestán debidamentebalanceados con susgastos. 
En una economía como la nuestra manejar bien las finanzas es de suma importancia, poseer una cultura del ahorro brinda seguridad y garantiza un mejor nivel de vida. Los estudiantes de la americana quienes se encaminan como futuros Administradores y Contadores deben tener total claridad sobre esta temática. Se puede decir, que es un factor fundamental o quizás la principal herramienta para que los individuos empiecen a cultivar buenos hábitos financieros; por lo tanto, dichas herramientas no deberían ser desconocidas para estos estudiantes quienes se enfilan en el mundo de las ciencias administrativas y contables. Su formación profesional va muy enfocada con factores económicos que nacen del ahorro y del sano manejo de las finanzas.

\section{Marco Teorico.}

En la teoría que realizó Modigliani (1986) sobre:

La Función de consumo, conocida como la "hipótesis del ciclo vital", dice que la mayor parte de las personas tiende a tener un nivel estable de consumo a lo largo de su vida. En determinados períodos los ingresos serán bajos, por lo que se endeudarán, mientras que, en otros, por ser éstos más altos, podrán ahorrar y prevenir para el futuro (párr.3).

Esta teoría es una vivencia natural; aportando a la presente investigación la claridad de que al iniciar la vida laboral los jóvenes atesoran ganancias de las cuales utilizan una gran parte para suplir necesidades básicas y algunos gustos extras, sin prepararse para el futuro mediante hábito del ahorro. Ya en la edad adulta y con madurez emocional empiezan a prepararse para los años venideros, adquiriendo bienes y destinando parte de sus ganancias para sobrevivir en una etapa donde seguramente no serán muy activos laboralmente.

La teoría del ingreso permanente para el consumo de Fierdman (1957) dice que el consumo y ahorro de un individuo no está directamente relacionado a la renta que obtiene al año, sino mas bien que se considera a través del tiempo. El aporte de Fierdman lleva a concluir que los individuos adoptan el hábito de ahorro cuando tienen ingresos superiores a los que normalmente obtienen en su cotidiano vivir, es decir, después de satisfacer las necesidades económicas básicas y gustos extras si queda algún porcentaje ese sería lo destinado al ahorro.
La hipótesis del ingreso relativo Duesenberry (1967) trata de que el individuo generalmente tiende a imitar ciertos patrones de la sociedad aún cuando sus ingresos están disminuyendo considerablemente. Se concluye que, los individuos en el momento en que sus ingresos empiezan a disminuir no variarán el consumo habitual y comenzarán a actuar seguidos por la moda adoptada por individuo de otro nivel social. La persona con el propósito de seguir en el mismo nivel social llegará a endeudarse y es claro que cuando los ingresos aumenten también aumentará el nivel de ahorro.

\section{Estado del Arte}

Las finanzas personas siempre están presenta en la vida de todos, abarcan en gran medida muchos aspectos en la vida cotidiana del ser humano, aspectos familiares, sociales, económicos y hasta psicológicos. Cada decisión financiera que se tome hoy tendrá un impacto positivo o negativo a lo largo de la vida económica de cada individuo. Por eso es de gran importancia que cada individuo maneje adecuadamente sus finanzas personales, y determine que cada decisión económica y financiera cuenta, incluso las más comunes y banales. Por consiguiente, al realizar este proyecto de investigación se tendrá en cuenta todos los aspectos que se consideren importantes en las decisiones financieras de los estudiantes de la CUA Montería, apoyado en las siguientes variables: Análisis, ahorro, Finanzas, estudiantes, empleo.

Todo tipo de comportamiento financiero y ahorrativo del ser humano como tal, conlleva siempre un análisis profundo en donde subyacen infinidades de características, bases y factores que explican las relaciones y decisiones financieras de cada individuo. Correa, Monsalve y Lopera (2017) en su investigación acerca del grado de conocimientos que poseen los Estudiantes de la Universidad de Buenaventura, Colombia, sobre sus finanzas personales y hábitos ahorrativos, refieren que la herramienta del Análisis es un elemento concluyente a la hora de indagar sobre las aptitudes financieras y económicas que poseen dichos estudiantes y como estos garantizan su propia estabilidad económica. El análisis permite comprender cada actitud, cada motivación, cada variabilidad que los individuos exponen frente a sus decisiones económicas, permitiendo llegar así a una conclusión. 
La anterior investigación se realizó tomado como objeto de muestra, el cuerpo estudiantil que está activo laboralmente, ofreciendo a la presente investigación la posibilidad de comparar los patrones de comportamiento financieros y económico que inciden en los estudiantes universitarios que laboran, también se puede analizar en nivel de educación financiera del estudiante en referencia a cada una de las bases y entendimientos que ha adquiridos durante todo su vida como estudiante y joven adulto. Dicho de otra manera, esa investigación será una guía que nos permitirá comprender las actitudes ahorrativas y hábitos financieros de los estudiantes asalariados frente a sus ingresos y gastos en un corto, mediano y largo plazo.

Así mismo, el tema del ahorro es otra herramienta que le permite al individuo obtener beneficios a corto, mediano y largo plazo, pues el ahorro es el único medio que garantizará que el individuo pueda ser capaz de sostenerse en el futuro y poder solventar sus de gastos imprevistos. Vanegas, Gómez \& White (2009) en su artículo sobre la capacidad de ahorro en estudiantes de formación superior afirman:

En cuanto a los posibles determinantes que pueda tener el ahorro de los estudiantes, aparece el ingreso y gasto o consumo, el primero puede ser definido como una variable no constante por las variaciones que se puedan presentar en el tiempo a causa de factores ajenos a los agentes económicos, mientras que el segundo se constituye como factor dependiente del ingreso y atiende a circunstancias que rodean a la persona, sus expectativas y la disciplina que tenga para el manejo o administración del gasto; en pocas palabras, se trata de un individuo que tiene una fuente de ingreso, con ciertos hábitos de consumo que le puedan facilitar o no el ahorro de una forma acertada (p. 3).

La investigación estudia los patrones de ingresos y gastos como determinantes del ahorro en los estudiantes del Tecnológico de Antioquia, Institución Universitaria. Se debe agregar que la anterior investigación aporta los posibles determinantes de la capacidad ahorrativa y financiera de un universitario promedio, además nos muestran una perspectiva de la situación actual de la población universitaria en lo que los hábitos de ahorro se refieren y permite visualizar el planteamiento de una propuesta financiera que principalmente incentive la cultura del ahorro.

Lo dicho hasta aquí supone la importancia del ahorro, sin embargo, éste dependerá mucho de cómo se manejan las finanzas personales, la aplicación en la economía del individuo y las decisiones económicas que se tomen. La educación financiera en edades temprana, también puede ser un factor primordial a la hora de crear buenos hábitos financieros; a menudo se piensa que la edad brinda la madurez económica necesaria para que el ser humano empiece a tener conciencia financiera y ahorrativa. Carangui, Garbay \& Valencia (2017) en su artículo investigativo, refieren que la edad de las personas predomina en la toma de sus decisiones financieras. La investigación tiene como propósito evidenciar la influencia de la edad en la toma de decisiones financieras de los individuos de una población en Ecuador, con la finalidad de determinar la prioridad en los gastos que realizan las personas de la localidad conforme avanza su edad. Concluyendo que:

La edad por sí sola no es un factor que influya directamente en la toma de decisiones de los individuos, profundizando la teoría neoclásica que indica que las personas tomamos decisiones influenciadas por el contexto, la teoría de racionalidad limitada expresa por su parte que los individuos poseemos limitaciones de conocimiento, enunciado que es sustentado en la investigación al determinar que los individuos no son capaces de equilibrar sus ingresos y gastos, menos propender al ahorro, forjando la necesidad de disminuir los niveles de analfabetismo financiero en la sociedad (p. 86).

Contextualizando, la relación de este antecedente con la presente investigación, está en analizar que existe más de una variable en la toma de decisiones económicas, que la edad por sí sola no es un factor que influya directamente en los hábitos y decisiones económicas de los estudiantes, aunque pueda ser un factor notorio, se tendría que analizar una variedad de factores como por ejemplo, el grado de educación financiera de la persona, si existen limitantes que impiden a la persona ahorrar, el tipo de ingreso que perciba el individuo, entre otros.

Con lo anterior expuesto, y poniendo en perspectiva este proyecto de investigación, se plantea que no es 
posible afirmar con precisión, que los estudiantes de la CUA montería, según el tipo de edad que tengan, así mismo van ahorrar.

Los estudiantes universitarios con carreras financieras, administrativas y contables, presentan bases que podrían ser de utilidad para sus conocimientos financieros sin son puesto en prácticas, sin embargo, no todos tendrán las mismas decisiones financiera, ni se enfrentarán a los mismos gastos. Reus, Garza y Reus (2015) en su artículo de investigación realizado en la Universidad de Guadalajara, México; habla acerca de ello, en dicha investigación se propone relacionar los gastos personales de los estudiantes de la carrera de Administración en su trayectoria escolar, mediante una Matriz de categorización que identifique los rublos de que es: -Urgente-No urgente- Importante y No importante en sus finanzas personales. Para llevar a cabo lo anterior, se contó también con instrumentos de recolección como las encuestas.

Este tipo de investigación arrojó fuertes bases que sirven de referente a nuestra propia investigación puesto que permitió analizar la conducta financiera de los estudiantes universitarios a la hora de ver como priorizan y clasifican sus gastos en lo que considerarían ellos son importantes y urgentes con respecto a sus finanzas personales. Dicho de otra manera, las decisiones financieras tomadas de manera consciente y asertiva le permiten al individuo una buena planeación económica y mejora su estabilidad financiera

\section{Metodología}

La metodología que se implementó fue de carácter descriptivo, que según Lerma (2009) "Su objetivo es describir el estado, las características, factores y procedimientos presentes en fenómenos y hechos que ocurren en forma natural, sin explicar las relaciones que se identifiquen” (p.63). Ya que, la investigación descriptiva se enfoca en conocer el fenómeno tal cual ocurre, y permite dar una explicación entre lo que está sucediendo y el medio que rodea dicho problema.

El enfoque de investigación es de tipo cualitativo que según Lerma (2009) "los investigadores desarrollan conceptos, intelecciones y comprensiones, partiendo pautas de los datos, y no recogiendo datos para evaluar modelos, hipótesis o teorías preconcebidas" (p.71). De lo anterior, se infiere que los resultados arrojados dependen de la interpretación que realice el investigador.

\section{Población y muestra}

La población son los estudiantes del programa de administración de empresas y contaduría pública de la Corporación Universitaria Americana, sede Montería, matriculados en el período de 2019-2.

Tabla 1

Matriculados en el periodo de 2019-2

\begin{tabular}{cccc}
20192 & $\begin{array}{c}\text { Nuev } \\
\text { os }\end{array}$ & $\begin{array}{c}\text { Antig } \\
\text { uos }\end{array}$ & $\begin{array}{c}\text { Tot } \\
\text { al }\end{array}$ \\
\hline $\begin{array}{c}\text { Administración De } \\
\text { Empresas }\end{array}$ & 20 & 123 & $\mathbf{3 5 3}$ \\
Contaduría Pública & 26 & 184 &
\end{tabular}

Fuente: Oficina admisiones y registros de la Corporación Universitaria Americana, 2019

Se tomó una muestra representativa de 136 estudiantes que se encuentran activos laboralmente.

Técnicas e instrumentos de recolección de datos

La técnica implementada es la encuesta, a través de un cuestionario estructurado con preguntas cerradas.

\section{Procedimiento detallado}

1. Cuantificación de la población activa laboralmente

2. Trabajo de campo (Realización de las encuestas de manera presencial)

3. Tabulación de encuestas

4. Análisis de resultados

5. Conclusiones

\section{Discusión.}

Los resultados arrojados muestran que el $60 \%$ de los encuestados están activos laboralmente, y un $40 \%$ depende económicamente de sus padres, teniendo encuenta lo aportado por Correa, Monsalve y Lopera (2017) contar con una fuente de ingreso le permite tener al individuo una actitud motivada, pues le permite tomar decisiones económicas de manera independiente y participar de manera activa en la economía del país. Otro resultado a tener en cuenta es que el $49 \%$ de los estudiantes encuestados no tienen personas a cargo, quiere decir, $13 \%$ tienen a cargo a sus padres, $18 \%$ tienen a cargo hijos, $7 \%$ tienen a cargo hermanos y 7\% están a cargo de sus esposas. Lo anterior hace referencia a que, con 
mayor número de personas a cargo, menos ingresos para ahorro tendrá la persona, esto se peude relacionar con la investigación realizada por Reus, Garza y Reus (2015) los cuales indican que la conducta financiera de los estudiantes universitarios depende en gran manera de lo que ellos consideran importante y urgente. Es así como sus decisiones financieras van a depender de las personas a cargo, tomando estás como importantes y urgentes, lo que puede dificultar una buena planeación financiera y por lo tanto la falta de organización les dificulta mantener una estabilidad financiera.

Por otro lado, a los encuestados se les preguntó si ellos presupuestaban sus gastos mensualmente, y el $60 \%$ manifestó que lo hacían frecuentemente, el $20 \%$ algunas veces y el otro $20 \%$ cuando se podía. Estos resultados demuestran que, aunque existe un leve conocimiento sobre planificación financiera, no existe con claridad los gastos mensuales en los que incurre cada individuo, lo anterior concuerda con lo aportado por Vanegas, Gómez y White (2009) quienes suponen que el ahorro depende en gran medida de como se manejan las finanzas personas y las decisiones que se tomen al respecto, creando habitos financieros saludables, donde la persona sea capaz de llevar una buena educación financiera que le permita mantener ingresos por encima de sus gastos, y no improvisar conrespecto a esta ultima variable, trayendo consigo el endeudamiento. Otro aspecto importante en la investigación es el rango de edades de los encuestados, y es que estos oscilan entre los 20 y 30 años, que según Carangui, Garbay y Valencia (2017) la edad incide mucho en la decisión de ahorrar, y consideran que esto es un factor muy importante que influye directamente en las decisones financeiras de los individuos, ya que según la teoría de la racionalidad limita, todos los individuos tienen limitaciones de conocimiento, por lo tanto no son capaces de nivelar sus ingresos y gastos, dificultando el ahorro y por ende aumentando los niveles de endeudamiento.

Finalmente, la investigación arrojó que los estudiantes, aunque conocen la importancia de ahorrar y tener una buena educación financiera en cuanto al manejo de sus gastos e ingresos, no realizan una planificación al respecto, ya que, tienen se dejan llevar por el día a día, la improvisación y por el consumo de cosas innecesarias, manteniendo en desequilibrio la balanza, y ocasionando que estás muchas veces se incline al lado negativo, dando como resultado el endeudamiento. Por lo tanto, el manejo de las finanzas personales de la población objeto de estudio radica en un bajo manejo y control del ahorro, al punto de no ahorrar y gastar más de lo que se gana. Lo que también influye, en las personas que tienen a cargo, en la edad y madurez, y por supuesto en los ingresos que reciben, que en muchos casos este último es inferior a los gastos.

\section{Conclusiones.}

Se puede concluir, que solo el $46 \%$ de la población objeto de estudio se encuentra activo laboralmente, quiere decir que el otro porcentaje depende económicamente de otra persona, la cual representa como papá, mamá o acudiente, que en muchos casos es un familiar cercano. Y al depender económicamente de alguien, dificulta la toma de decisiones financeiras, ya que necesariamente requieren autorización de su acudiente para hacer uso de los recursos económicos dispuestos para los gastos del estudiante.

Así mismo, se concluye el rango de edad de los que se encuentran activos laboralmente es de los 20 a los 30 años, los cuales se evidencia ya están en su mayoría de edad, y por lo tanto, pueden decir que hacer con sus área financiera, sin embargo, la edad no indica madurez, pues está radica más en la experiencia, y en este caso, es la experiencia en el manejo del dinero.

El $100 \%$ de los encuestados tiene claridad sobre la cultura del ahorro, conocen la importancia, saben a ciencia cierta que ahorrar trae muchos beneficios, sin embargo, a pesar de conocer todo esto, son pocos los que lo aplican realmente en su vida, trayendo como resultado muchos inconvenientes económicos, al punto de generar deudas que en muchas ocasiones no pueden ser solventadas. Ya que, al no destinar un porcentaje de los ingresos al ahorro, no van a contar con un deposito a su favor para cualquier emergencia, percance o situación no planeada que requiera dinero. Esto se debe, a una falta de planificación financiera.

Son muchos los factores que inciden en los estu- 
diantes al momento de manejar sus finanzas y ahorrar, uno de ellos es la edad, pues según la teoría de la racionalidad nuestro cerebro es limitado, lo que dificulta tener una madurez para tomar decisiones.

Otro factor que incide en el correcto manejo de las finanzas, es diferenciar entre los gastos urgentes y los gastos importantes, al no tener claridad sobre esto, el individuo tiende a toamr decisiones erróneamente que le pueden causar un endeudamiento innecesario.

La dependencia económica a los individuos encuestados es otro factor que influye en la correcta toma de decisiones, pues al tener varias personas a su cargo es mayor el peso económico que tiene que afrontar el estudiante. Lo que puede dificultar el buen manejo de las finanzas sino se hace una planeación financiera correcta.

En definitiva, el manejo de las finanzas personales es algo que afecta a toda la población, no hay distinción de raza, edad o estrato socieoeconomico, ya que desde el nacimiento se empieza a ser actor de la economía del país.

\section{Referencias}

Carangui, P., Garbay, J. y Valencia, B. (2017). Finanzas personales: la influencia de la edad en la toma de decisiones financieras. Revista Killkana Sociales, 1(3), 81-88. Recuperado de https:// dialnet.unirioja.es/servlet/articulo?codigo $=6297480$

Correa, V., Monsalve, A. y Lopera. J. (2017). Conocimiento del Manejo de las Finanzas Personales en los estudiantes asalariados de la Facultad de Ciencias Empresariales de la Universidad San Buenaventura Medellín. (Tesis de Pregrado). Universidad de San Buenaventura, Medellín, Colombia. Recuperado de http://webcache.googleusercontent.com/ search?q=cache:FM6rPXf-oNEJ:www.bibliotecadigital.usb. edu.co/bitstream/10819/5669/1/Conocimiento_Manejo_ Finanzas_Correa_2017.pdf $+\& \mathrm{~cd}=1 \& \mathrm{chl}=\mathrm{es} \& \mathrm{ct}=\mathrm{clnk} \& \mathrm{gl}=\mathrm{co}$

Duesenberry, J. (1967). Renta, Ahorro y Teoría del Comportamiento del Consumidor. Madrid, España: Alianza Editorial, S. A.
Friedman, M. (1957). A Theory of the Consumption Function. Nueva Jersey: Estados Unidos: Alianza Universidad.

Lerma González, H. D. (2009). Metodología de la investigación (4ta Edición ed.). Bogotá, Colombia: Eco Ediciones

Méndez, H. (2015). Estrés en adolescentes que estudian y trabajan. (Tesis de Pregrado). Universidad Rafael Landívar, Guatemala. Recuperado de: http://recursosbiblio.url.edu.gt/ tesiseortiz/2015/05/22/Mendez-Hugo.pdf

Modigliani, f. (1986). El ciclo vital, el ahorro individual y la riqueza de las naciones. Papeles de Economía Española, (28), 297-315.

Portafolio. (7 de Marzo de 2016). Universitarios Colombianos, malos en finanzas personales. Recuperado de https://www.portafolio. co/mis-finanzas/ahorro/universitarios-colombianos-malosfinanzas-personales-491250

Redy, R., Bruhn, M., \& Tan, C. (2013). Capacidades Financieras en Colombia: Resultados de la encuesta nacional sobre comportamientos, actitudes y conocimientos financieros. Banco Internacional de Reconstrucción y Fomento/Banco Mundial. Recuperado de: https://www.bancomundial.org/ content/dam/Worldbank/document/LAC/Capacidades $\% 20$ Financieras\%20en\%20Colombia.pdf

Reus,N.,Reus,T.yGarza,M.,(2015).Matrizdeclasificacióndegastos deacuerdoalaimportanciaqueledanlosalumnosensusfinanzas personales. Revista Iberoamericana de Producción Académica y Gestión Educativa, (2). 1-15. Recuperado de http://repositorio. cualtos.udg.mx:8080/jspui/bitstream/123456789/299/1/ Matriz\%20de\%20clasificacion\%20de\%20gastos.pdf

Vanegas, Gómez \& White. (2009). Capacidad de ahorro en estudiantes de educación superior. Un estudio empírico en el Tecnológico de Antioquia. Revista En-Contexto, 1(1), 123-139. Recuperado de https://ojs.tdea.edu.co/index.php/encontexto/ article/view/50 